Majorana A1., Merlo J1., Bardellini E1.

1Department of Medical and Surgical Specialities, Radiological Sciences and Public Health, Dental School, University of Brescia, Brescia, Italy

\title{
Pilot study on dysphagia in children undergoing chemotherapy: the COMEDY pattern
}

\begin{abstract}
AIM
The aim of this study was to evaluate the association between non-erosive reflux disease (NERD) and a specific oral mucosal traumatic alteration in children with dysphagia undergoing chemotherapy.
\end{abstract}

\section{METHODS}

Children manifesting both dysphagia and chemotherapyrelated oral mucositis $(\mathrm{OM})$ within the last year were considered for this study. Oral lesions characterized by a traumatic appearance i.e. milky opalescent mucosa, edema and teeth imprints were selected. Every patient received an otolaryngology consultation to evaluate NERD related changes. Since as defensive reaction to swallowing the children clench, patients were instructed on how to avoid it and, in case of indirect signs of reflux, were treated with sodium alginates and proton pump inhibitors. A hyaluronic acid topical therapy was also recommended. Oral mucosal pain was evaluated through the Visual Analogue Scale (VAS) at T0 and after a week (T1).

\section{RESULTS}

Out of 54 patients with OM, 8 patients (14.8\%) were included in the study. All children presented indirect signs of NERD and were consequently treated, besides receiving anti-clenching instructions. They all physically manifested closed-off expression (closed eyes and shoulder, limited interaction) due to dysphagia discomfort. After therapy, at T1 they demonstrated a significant improvement in pain and the resolution of oral lesions.
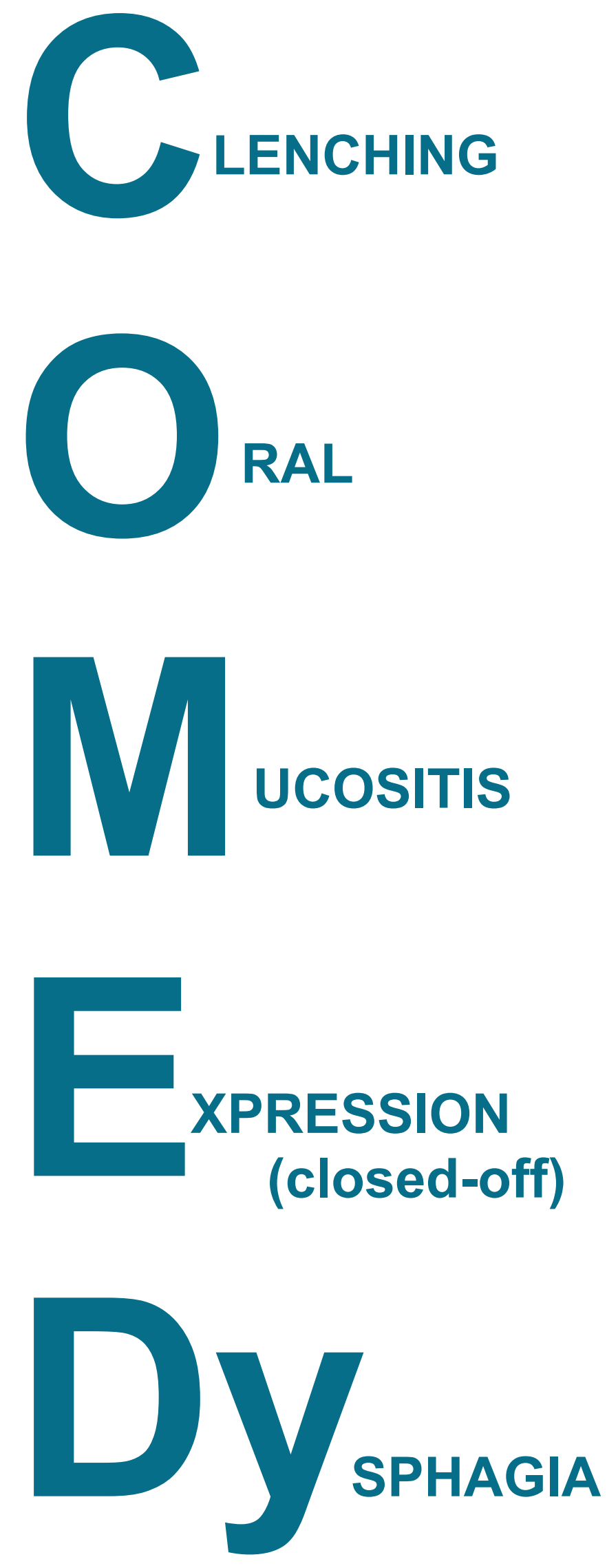
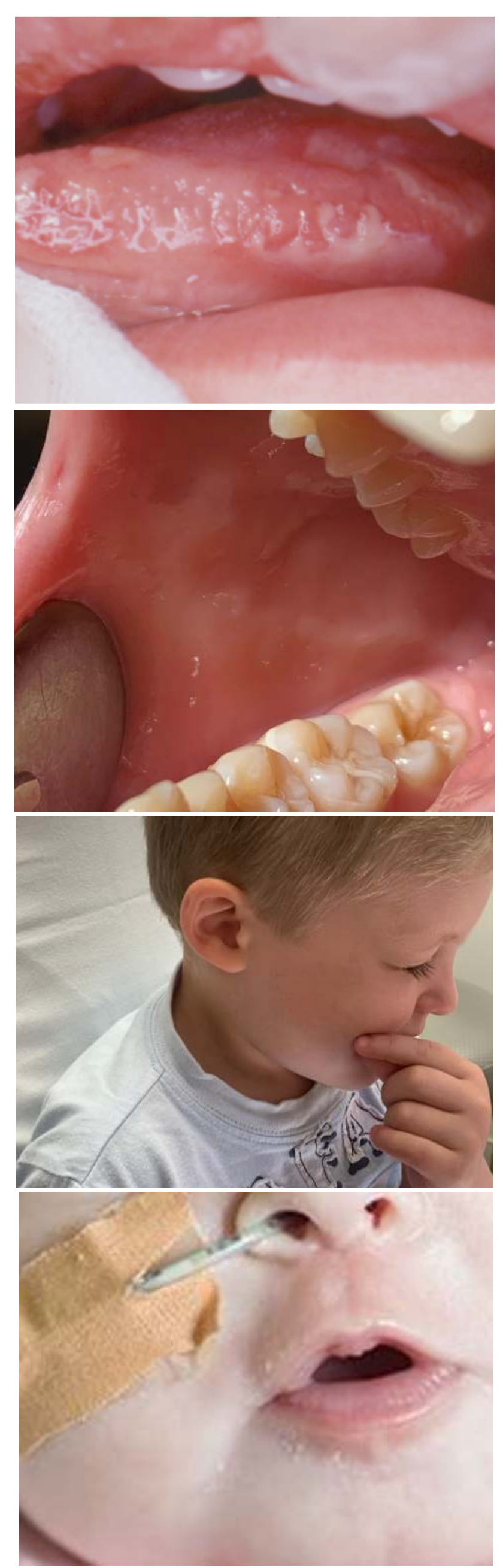

\section{CONCLUSIONS}

All these elements configure a whole nosological entity, which includes Clenching, OM, closed-off Expression, and Dysphagia, summarized in the acronym "COMEDY." Recognizing the COMEDY pattern in children undergoing chemotherapy could help to direct treatment toward a combination of conventional NERD therapy and a behavioral approach, besides topical treatment, to resolve oral mucosal lesions. 